\title{
A vibração torácica na fisioterapia respiratória de recém-nascidos causa dor?
}

\author{
Does chest vibration during respiratory physiotherapy in neonates cause pain?
}

\begin{abstract}
Fernanda de Cordoba Lanza', Andrea Hye K. Kim², Juliana Leite Silva², Amanda Vasconcelos ${ }^{2}$, Sabrina Pinheiro Tsopanoglou ${ }^{3}$
\end{abstract}
\section{RESUMO}

Objetivo: Avaliar a dor em recém-nascidos pré-termo (RNPT) submetidos à fisioterapia respiratória em Unidade de Terapia Intensiva Neonatal.

Métodos: Estudo transversal realizado com recém-nascidos prematuros, com indicação de fisioterapia respiratória, em respiração espontânea. Foi aplicada uma técnica de vibração torácica com a mão do terapeuta realizando pequenas oscilações sobre o tórax do paciente e feita a avaliação da frequência cardíaca (FC), frequência respiratória (FR), saturação de pulso de oxigênio $\left(\mathrm{SpO}_{2}\right)$ e do Sistema de Codificação da Atividade Facial Neonatal (NFCS) para a avaliação da dor antes, durante, imediatamente após (pós-i) e 30 minutos após (pós-30) a vibração no tórax do paciente. Utilizou-se a análise de variância para medidas repetidas para comparação entre as fases, considerando-se significante $p<0,05$.

Resultados: Foram avaliados 13 RNPT, com média de idade gestacional 32,5 $\pm 2,0$ semanas e peso de nascimento $1830 \pm 442 \mathrm{~g}$. Não foi observada pontuação de dor durante toda a avaliação: pré: $0,5 \pm 1,7$; durante: $1,5 \pm 1,4$; pós-i: $1,0 \pm 1,3$; pós-30: $0 \pm 0,3$, porém houve diferença estatisticamente significativa entre a fase pós-i e pós-30 na escala NFCS $(p<0,05)$. A FC variou de 120 a 150 bpm, a $\mathrm{SpO}_{2}$ permaneceu acima de $95 \%$, e a FR entre 40 e $62 \mathrm{rpm}$, sem diferenças entre os períodos.

Conclusões: Neste grupo de pacientes prematuros internados em UTI neonatal e submetidos à realização de fisioterapia respiratória pela técnica de vibração torácica, não foram observadas alterações fisiológicas e comportamentais de dor.

Palavras-chave: dor; fisioterapia; prematuro; Unidade de Terapia Intensiva Neonatal.

Instituição: Centro Universitário São Camilo, São Paulo, SP, Brasil

'Doutora em Ciências pela Disciplina de Alergia, Imunologia Clínica e Reumatologia do Departamento de Pediatria da Universidade Federal de São Paulo (Unifesp); docente do Centro Universitário São Camilo, São Paulo, SP, Brasil

${ }^{2}$ Fisioterapeuta graduada pelo Centro Universitário São Camilo, São Paulo, $\mathrm{SP}$, Brasil

${ }^{3}$ Especialista em Fisioterapia Respiratória pela Unifesp; docente do Centro Universitário São Camilo, São Paulo, SP, Brasil

\section{ABSTRACT}

Objective: To observe pain score during chest physiotherapy (CP) in preterm newborns (PTNB) assisted in a Neonatal Intensive Care Unit.

Methods: A cross-sectional study was carried out with PTNB with spontaneous breathing that needed respiratory physiotherapy. A vibration technique was employed, with the hand of the therapist applying oscillations on the patients' thorax. The following variables were assessed: heart rate $(\mathrm{HR})$, respiratory rate $(\mathrm{RR})$ and oxygen saturation ( $\mathrm{O}_{2}$ Sat), as well as the Neonatal Facial Coding System (NFCS) to analyze pain before (pre), during, just after and 30 minutes after the thoracic vibration technique. Repeated measures analysis of variance was applied to analyze the protocol phases, being significant $p<0.05$.

Results: Thirteen PTNB were assessed. The mean gestational age was $32.5 \pm 2.0$ weeks, and the birth weight was $1830 \pm 442 \mathrm{~g}$. No pain was observed during the evaluation: "pre": 0.5 \pm 1.7 ; "during": $1.5 \pm 1.4$; “just after": $1.0 \pm 1.3 ;$ "30 min after": $0 \pm 0.3$, but there was difference in the NFCS scores between the periods "just after" and "30 minutes after" $(p<0.05)$. The HR varied between 120 and $150 \mathrm{bpm}, \mathrm{O}_{2}$ Sat levels remained over $95 \%$, and RR oscillated from 40 to $62 \mathrm{mpm}$, without differences between periods.

Conclusions: Preterm patients spontaneously breathing and submitted to respiratory physiotherapy by thoracic vibration, no evidence of physiological or behavioral pain indicators were observed.

Key-words: pain; physical therapy; infant, premature; Intensive Care Unit, Neonatal.

Endereço para correspondência:

Fernanda de Cordoba Lanza

Rua Estado de Israel, 465, apto. 23 - Vila Clementino

CEP 04022-001 - São Paulo/SP

E-mail: fclanza@uol.com.br

Conflito de interesse: nada a declarar

Recebido em: 20/4/2009

Aprovado em: 6/8/2009 


\section{Introdução}

Até a década de 1970, acreditava-se que recém-nascidos não sentiam dor, pois seu sistema neurológico ainda seria imaturo e, por isso, esses pacientes estariam "protegidos"(1-5). Atualmente, sabe-se que os recém-nascidos pré-termo são capazes de sentir dor e, além disso, são mais sensíveis a ela do que crianças maiores ou adultos ${ }^{(6-8)}$. As experiências dolorosas ou repetidas vivenciadas nos períodos peri e pósnatal podem causar efeitos neurobiológicos prolongados na estrutura cerebral e nas futuras reações comportamentais da criança, como déficits cognitivos e motores diagnosticados na idade pré-escolar e escolar ${ }^{(9-13)}$.

Para avaliar a dor do neonato, que não pode expressá-la por meio de palavras e para atenuar a subjetividade das medidas comportamentais de dor e facilitar sua aplicação na prática clínica, foram desenvolvidas escalas de avaliação da dor uni ou multidimensionais. Dentre as primeiras, destaca-se o Sistema de Codificação da Atividade Facial (NFCS) ${ }^{(14-18)}$. Essa escala comportamental avalia a presença ou ausência de movimentos faciais, que constituem a resposta mais específica aos procedimentos dolorosos agudos exibida pelos recém-nascidos.

Os prematuros frequentemente apresentam doenças respiratórias e a abordagem fisioterapêutica faz parte do tratamento proposto nas Unidades de Terapia Intensiva Neonatais. Nos casos em que se objetiva a remoção de secreção do trato respiratório inferior, podem ser empregadas técnicas de higiene brônquica, como a vibração, a drenagem postural e a aspiração. A ênfase é direcionada às técnicas passivas e/ou de posicionamento para preservar a estrutura respiratória, evitando ou minimizando a dor e a manipulação excessiva ${ }^{(19-23)}$. A técnica de vibração auxilia na mudança das características da secreção, facilitando a sua remoção ${ }^{(23,24)}$. Entretanto, ainda não está claro se técnicas de fisioterapia desencadeiam dor no prematuro.

O objetivo deste estudo foi avaliar os parâmetros fisiológicos e comportamentais de dor em recém-nascidos pré-termo submetidos à vibração torácica para fisioterapia respiratória.

\section{Métodos}

O projeto foi aprovado pelo Comitê de Ética em Pesquisa do Centro Universitário São Camilo e solicitou-se a assinatura do termo de consentimento livre e esclarecido pelos responsáveis legais do recém-nascido. Trata-se de um estudo transversal cujos critérios de inclusão eram: recém-nascidos em respiração espontânea em ar ambiente ou oxigenoterapia inalatória, com idade gestacional entre 30 e 36 semanas, internados em unidade de terapia intensiva, com indicação de fisioterapia respiratória por meio da técnica de vibração torácica para higiene brônquica. Os critérios de exclusão foram: realização de outra técnica de higiene brônquica, uso de sedativos e/ou analgésicos nas últimas 72 horas, osteopenia da prematuridade, uso de ventilação mecânica invasiva ou não-invasiva, presença de dreno torácico, dreno abdominal ou cateter umbilical, presença de qualquer incisão torácica/abdominal, manipulação 30 minutos antes da coleta de dados, diagnóstico de hemorragia peri-intraventricular graus III e IV ou qualquer outra lesão no sistema nervoso central.

Uma vez incluídos, os recém-nascidos foram submetidos à fisioterapia vibratória e à avaliação da dor por meio de parâmetros fisiológicos e comportamentais descritos abaixo, que foi aplicada nos seguintes momentos: antes do procedimento (Repouso), cinco minutos após o início da fisioterapia (Tratamento), imediatamente após o término da vibração torácica (Pós-i) e 30 minutos após o término da fisioterapia (Pós-30).

$\mathrm{Na}$ fase inicial, o recém-nascido era posicionado em decúbito dorsal e, em seguida, era avaliada a frequência cardíaca (FC) e a saturação de oxigênio $\left(\mathrm{SpO}_{2}\right)$ com oxímetro de pulso da marca Dixtal ${ }^{\circledR}$. Além disso, verificava-se a frequência respiratória (FR), contando-se as incursões torácicas durante um minuto. Realizava-se, então, a avaliação da dor pela escala NFCS definida pela presença ou ausência de oito características faciais: fronte saliente (abaulamento e presença de sulcos acima de e entre as sobrancelhas), olhos espremidos (compressão total ou parcial da fenda palpebral), sulco nasolabial aprofundado (aprofundamento do sulco que se inicia em volta das narinas e se dirige à boca), lábios entreabertos (qualquer abertura dos lábios), boca esticada (vertical, com o abaixamento da mandíbula, ou horizontal, com estiramento das comissuras labiais), lábios franzidos (parecem estar emitindo um som de “ú”), língua tensa (protusa, esticada e com as bordas tensas) e tremor do queixo ${ }^{(15)}$. Para cada itens citado, quando presente, atribuiu-se 1 ponto, quando ausente, 0 ponto. O escore máximo foi de 8 pontos e considerou-se a presença de dor quando a pontuação foi superior a $3^{(16)}$. Os pacientes avaliados permaneceram 60 minutos em repouse antes de iniciar o protocolo.

$\mathrm{Na}$ fase tratamento, realizava-se a fisioterapia respiratória sempre pelo mesmo fisioterapeuta. A técnica de vibração foi feita pelo terapeuta, com a mão posicionada sobre o tórax do paciente e contração isométrica de antebraço e braço. A vibração foi aplicada bilateralmente sem se associar à drenagem postural. Após cinco minutos do início da terapia, foi feita 
nova avaliação da $\mathrm{FC}, \mathrm{SpO}_{2}$ e FR e da dor pela escala NFCS, sem que houvesse interrupção do tratamento. $\mathrm{O}$ tratamento não ultrapassava 20 minutos, como preconizado para este grupo de pacientes.

Durante a fase pós-tratamento imediato (pós-i) e 30 minutos pós-tratamento (pós-30), aplicou-se a escala NFCS acompanhada da avaliação da FC, $\mathrm{SpO}_{2}$ e FR.

O cálculo amostral foi realizado levando-se em consideração que a técnica utilizada poderia mudar em 1,5 pontos a escala de dor, com desvio padrão de 1 , poder de $80 \%$ e alfa de $5 \%$, resultando em oito indivíduos. Foi realizada a análise de variância para medidas repetidas para a comparação da escala NFCS entre as quatro fases de observação do protocolo (pré-tratamento, durante o tratamento, pós-i, pós-30), complementada pelo teste Tukey-Kramer para localizar as diferenças. Os dados foram expressados em média e desvio padrão. Considerou-se significante $p<0,05$.

\section{Resultados}

Foram avaliados 16 recém-nascidos prematuros na UTI do Hospital Infantil Cândido Fontoura, no período de agosto a dezembro de 2006. Desses, um paciente foi excluído devido a problemas para monitorizar FC, FR e SpO e dois, devido à necessidade de aspiração traqueal após a

Tabela 1 - Dados demográficos dos 13 pacientes estudados

\begin{tabular}{lrc}
\hline & Mediana & $\begin{array}{c}\text { Intervalo } \\
\text { interquartil }\end{array}$ \\
\hline IG (semanas) & 32 & $30-35$ \\
Peso ao nascer $(\mathrm{g})$ & 1830 & $1100-2579$ \\
Apgar $1^{\circ}$ minuto & 6 & $4-8$ \\
Apgar 5 $5^{\circ}$ minutos & 7 & $6-9$ \\
Idade na avaliação (dias) & 16 & $10-23$ \\
IG na avaliação (semanas) & 35 & $32-38$ \\
Peso na avaliação $(\mathrm{g})$ & 2000 & $1350-2559$ \\
Procedimentos/dia $(\mathrm{n})$ & 60 & $23-90$ \\
\hline
\end{tabular}

IG: idade gestacional. técnica de vibração. Portanto, para a avaliação final foram incluídos 13 RNPT.

Dos neonatos estudados, $61 \%$ eram do sexo masculino. A média de idade gestacional foi $32 \pm 2$ semanas, com peso de nascimento de $1830 \pm 442 \mathrm{~g}$. Os recém-nascidos foram submetidos a $60 \pm 21$ procedimentos/dia antes de serem incluídos no estudo. Os dados demográficos estão descritos na Tabela 1.

Em relação à escala de dor avaliada, observou-se diferença significante entre a fase tratamento e 30 minutos após término do tratamento $(p<0,05)$, entretanto, em nenhum momento o escore médio da escala indicou a presença de dor (Tabela 2). Não houve alteração significativa das variáveis fisiológicas avaliadas ( $\mathrm{FC}, \mathrm{FR}$ e $\mathrm{SpO}_{2}$ ) durante as fases do estudo (Tabela 2).

O tempo total de realização da terapia respiratória nos pacientes avaliados foi de $17 \pm 3,2$ minutos.

\section{Discussão}

Após terem sido estudados 13 RNPT submetidos à vibração como técnica de fisioterapia respiratória na UTI neonatal, não foi observada pontuação de dor durante a manipulação avaliada pela escala NFCS e também não foram encontradas variações significativas nos parâmetros fisiológicos.

A avaliação da dor no período neonatal, segundo Guinsburg et al ${ }^{(17)}$, baseia-se em modificações de parâmetros fisiológicos ou comportamentais observados antes e depois de um estímulo doloroso. As medidas fisiológicas para avaliação da dor mais utilizadas na prática clínica são a frequência cardíaca, a frequência respiratória, a pressão arterial e dosagem de hormônios de estresse, mas algumas escalas específicas têm sido aplicadas para esta finalidade, entre elas a NFCS. As modificações da mímica facial após estímulo doloroso (punção de calcanhar) são rápidas e, um a três minutos após o estímulo doloroso, a mímica de dor já não apresenta sua expressão plena ${ }^{(25,26)}$. Neste estudo, foram avaliadas a frequência cardíaca, a frequência res-

Tabela 2 - Dados da escala de Dor (NFCS) e parâmetros fisiológicos

\begin{tabular}{lcccc}
\hline & Repouso & Tratamento & Pós-imediato & Pós 30 minutos \\
\hline NFCS & $0,5 \pm 1,7$ & $1,5 \pm 1,4$ & $1,0 \pm 1,3$ & $0 \pm 0,3$ \\
FC $(\mathrm{bpm})$ & $142 \pm 16$ & $146 \pm 17$ & $154 \pm 20$ & $142 \pm 15$ \\
FR $($ rpm) & $54 \pm 9$ & $56 \pm 9$ & $54 \pm 10$ & $53 \pm 11$ \\
SpO2 $(\%)$ & $97 \pm 1$ & $97 \pm 1$ & $97 \pm 2$ & $97 \pm 2$ \\
\hline
\end{tabular}

${ }^{*} p<0,05$ (fase tratamento versus após 30 minutos de tratamento).

NFCS: Sistema de Codificação da Atividade Facial Neonatal; FC: frequência cardíaca; FR: frequência respiratória; SpO2: saturação de pulso de oxigênio. 
piratória, a saturação de oxigênio e a mímica facial pela escala NFCS antes, durante, imediatamente após e 30 minutos após o término da fisioterapia respiratória para identificar possíveis alterações na escala de dor ou nos parâmetros fisiológicos. Não foram realizados exames laboratoriais, como dosagem de hormônios, pois a proposta foi realizar uma avaliação da dor que a identificasse rapidamente, para que se pudesse fazer uma intervenção imediata.

Os prematuros avaliados apresentaram alteração no escore da escala NFCS entre a fase tratamento e 30 minutos após a realização da fisioterapia. Embora tenha havido diferença estatisticamente significativa entre estas fases, os valores observados não ultrapassaram a pontuação 3 , limite considerado de dor. Sabe-se que as alterações na face do prematuro após um período de dor ou estresse perduram por poucos minutos, revertendo rapidamente ${ }^{(17)}$. Este dado foi constatado em nosso estudo, pois na fase pós-30 o valor da NFCS era semelhante ao valor da fase inicial.

O tratamento fisioterapêutico realizado foi um procedimento não-invasivo, o que justifica a não-observação da dor. Alguns estudos mostraram que procedimentos não-invasivos (fricção do calcanhar), comparados a procedimentos invasivos (punção do calcanhar), não causam dor ${ }^{(5,16)}$, mas isso provavelmente depende do número prévio de exposições à dor e da sensibilização das vias periféricas e da medula espinal à estimulação nociceptiva. A média do número de procedimentos prévios realizados nos pacientes estudados foi 59,6. As manipulações realizadas previamente neste grupo provavelmente sensibilizaram as vias nociceptivas, entretanto, não da mesma forma relatada para os bebês mais imaturos, que podem ser submetidos a um número maior de procedimentos dolorosos por dia e têm uma imaturidade ainda maior dos sistemas moduladores da dor. Desta forma, não se pode extrapolar os dados aqui obtidos para os prematuros com idade gestacional abaixo de 30 semanas.

$\mathrm{O}$ recém-nascido pré-termo submetido ao estresse ou à dor apresenta alterações hemodinâmicas, ventilatórias e metabólicas ${ }^{(2,5)}$. A dor sentida pelos prematuros está associa- da à alteração da FC e FR por liberação de neurotransmissores. Entretanto a inibição efetiva deste estado é imatura, o que perpetuará a dor, resultando em alterações em curto e longo prazo no prematuro. Dessa forma, torna-se importante avaliar a dor do paciente e, diante da sua presença, interromper a manipulação que está causando a dor. Não se observaram, no presente estudo, alterações de parâmetros fisiológicas (FC, FR e $\mathrm{SpO}_{2}$ ), os quais permaneceram dentro dos limites de normalidade para a idade, sugerindo que os prematuros avaliados não apresentaram dor durante a vibração do tórax.

Em casos de procedimentos dolorosos ou estressantes, recomenda-se o uso de analgésicos para evitar as consequências da dor ${ }^{(18,27)}$. Como, em nosso estudo, não foi observada dor e/ou alterações nos parâmetros fisiológicos, não se fez necessário aplicar medicações para sua atenuação.

Um curto relato na literatura faz menção à avaliação das técnicas de fisioterapia e ao desencadeamento de dor. Os autores observaram não haver diferença quanto à presença de dor antes e após a fisioterapia respiratória, mas não há relato de qual técnica foi utilizada. Entretanto, houve presença de dor durante e logo após a aspiração traqueal, técnica invasiva por vezes necessária para eliminar a secreção das vias aéreas do prematuro e que pôde ser realizada por diversos profissionais da saúde ${ }^{(28)}$. Sendo assim, as manobras para eliminar a secreção pulmonar no prematuro devem ser rigorosamente escolhidas para que não haja efeito colateral à sua utilização e, caso sejam necessárias, é preciso ter em mãos recursos para amenizar a dor.

Conclui-se que a técnica de vibração para a fisioterapia respiratória de recém-nascidos moderadamente prematuros que não precisam de ventilação mecânica não provoca dor ou alterações nos parâmetros fisiológicos como $\mathrm{FC}, \mathrm{FR}$ e $\mathrm{SpO}_{2}$.

\section{Agradecimentos}

Agradecemos ao Centro Universitário São Camilo, São Paulo (SP).

\section{Referências bibliográficas}

1. Anand KJS, Grunau RE, Oberlander TF. Developmental character and longterm consequences of pain in infants and children. Child Adolesc Psychiatric Clin N Am 1997;6:703-24.

2. Dinerstein A, Brundi M. El dolor en el recién nacido prematuro. Rev Hosp Matern Infant Ramon Sarda 1998;17:146-54.
3. Anand KJ, Carr DB. The neuroanatomy, neurophysiology, and neurochemistry of pain, stress, and analgesia in newborns and children. Pediatr Clin N Am 1989;36:795-822.

4. Espinosa AG, Mendonza AR. Dolor en recién nacidos. Acta Pediatr Méx 1997;18:94-5. 
5. Stevens B, Yamada J, Ohlsson A. Sucrose for analgesia in newborn infants undergoing painful procedures. Cochrane Database Syst Re 2004;(3):CD001069.

6. Guinsburg R. Avaliação e tratamento da dor no recém-nascido. J Ped (RJ) 1999;75:149-60.

7. Johnston CC, Stevens BJ. Experience in a neonatal intensive care unit affects pain response. Pediatrics 1996;98:925-30.

8. Abu-Saad HH, Bours GJ, Stevens B, Hamers JP. Assessment of pain in the neonates. Semin Perinatol 1998;22:402-16.

9. Okada M, Texeira MJ, Tengan SK, Moraes LO. Desenvolvimento do sistema nociceptivo e supressor da dor. Rev Med (São Paulo) 2001;80:78-93.

10. Holsti L, Grunau RE, Whifield MF, Oberlander TF, Lindh V. Behavioral responses to pain are heightened after clustered care in preterm infants born between 30 and 32 weeks gestational age. Clin J Pain 2006;22:757-64.

11. Leslie AT, Guinsburg R, Mello LE, Covolan L. Repetitive nociceptive stimuli in newborn rats do not alter the hippocampal neurogenesis. Pediatr Res 2008;63:154-7.

12. Craig KD, Whitfield M, Grunau RVE. Pain in the preterm neonate: behavioral and physiological indices. Pain 1993;52:287-99.

13. Anand KJ. Clinical importance of pain and stress in preterm neonates. Biol Neonate 1998;73:1-9.

14. Bergamasco NH. Expressão facial como acesso à consciência do recémnascido. Psicol USP 1997;8:275-86.

15. Grunau RV, Craig KD. Pain expression in neonates: facial action and cry. Pain 1987;28:395-410.

16. Grunau RV, Oberlander T, Holsti L, Whitfield MF. Bedside application of the Neonatal Facial Coding System in pain assessment of premature neonates. Pain 1998;76:277-86.

17. Guinsburg R, Balda RC, Berenguel RC, Almeida FB, Tonelloto J, Santos AM et al. Aplicação das escalas comportamentais para a avaliação da dor em recém-nascidos. J Pediatr (Rio J) 1997;73:411-8.

18. No authors listed. Prevention and management of pain and stress in the neonate. American Academy of Pediatrics. Committee on Fetus and Newborn.
Committee on Drugs. Section on Anesthesiology. Section on Surgery. Canadian Paediatric Society. Fetus and Newborn Committee. Pediatrics 2000;105: 454-61.

19. Haddad ER, Costa LC, Negrini F, Sampaio LM. Abordagens fisioterapêuticas para a remoção de secreções das vias aéreas em recém-nascidos: relato de caso. Pediatria (São Paulo) 2006;28:135-40.

20. Flenady VJ, Gray PH. Chest physiotherapy for preventing morbidity in babies being extubated from mechanical ventilation. Cochrane Database Syst Rev 2002:CD000283.

21. Antunes LC, Rugolo LM, Crocci AJ. Effect of preterm infant position on weaning from mechanical ventilation. J Pediatr (Rio J) 2003;79:239-44.

22. Perrotta C, Ortiz Z, Roqué FM. Chest physiotherapy for acute bronchiolitis in paediatric patients between 0 and 24 months old. Cochrane Database of Systematic Reviews 2007:CD004873.

23. Hough JL, Flenady V, Johnston L, Woodgate PG. Chest physiotherapy for reducing respiratory morbidity in infants requiring ventilatory support. Cochrane Database of Systematic Reviews 2008:CD006445.

24. Freitas A. Particularidades sobre a assistência fisioterapêutica respiratória em pediatria e neonatologia: Manobras de higiene brônquica. In: Sarmento GJV. Fisioterapia respiratória no paciente crítico: rotinas clínicas. São Paulo: Manole; 2005, p. 376-84.

25. Gibbins S, Stevens B, McGrath PJ, Yamada J, Beyene J, Breau L et al Comparison of pain responses in infants of different gestational ages. Neonatology 2008;93:10-8.

26. Pereira AL, Guinsburg R, de Almeida MF, Monteiro AC, dos Santos AM, Kopelman BI. Validity of behavioral and physiologic parameters for acute pain assessment of term newborn infants. Sao Paulo Med J 1999;117: $72-80$

27. Anand KJ, International Evidence-Based Group for Neonatal Pain. Consensus statement for the prevention and management of pain in the newborn. Arch Pediatr Adolesc Med 2001;155:173-80.

28. Falcão MC. Fisioterapia respiratória no período neonatal. Carta ao editor. Pediatria (São Paulo) 2006;28:211-2. 